\title{
The Relationship between Cognitive Impairment and Coronary Artery Disease in Middle-aged Adults
}

\author{
Nasser T. Balbaid ${ }^{1}$, Ahmad Al-Dawalibi ${ }^{1}$, Abdullah M. Khattab ${ }^{1}$, Fahad Al-Saqr ${ }^{1}$, Abdulrahman \\ AbuSittah $^{1}$, Sami Alqarni ${ }^{1}$, Syed Habib ${ }^{1}$, Muhammad Iqbal ${ }^{1}$, Shahid Bashir ${ }^{2}$ \\ 1. Physiology, College of Medicine, King Saud University, Riyadh, SAU 2. Neurophysiology, Neuroscience Center, King \\ Fahad Specialist Hospital, Dammam, SAU
}

Corresponding author: Shahid Bashir, shahidbashir101@hotmail.com

\section{Abstract \\ Background}

Cognitive impairment is a phenomenon that appears late in many diseases. Many clinicians do not seriously consider cognitive impairment until there has been significant deterioration over time. Cognitive function can be assessed using the Cambridge Neuropsychological Test Automated Battery (CANTAB).

\section{Methods}

Using an observational case-control study design, we examined the relationship between coronary artery disease (CAD) and cognitive impairment. Participants (57 patients with CAD and 60 healthy controls; age: 30-60 years) were recruited and sampled using a non-probability quota sampling technique. Selfadministered questionnaires were used to collect data about participants' demographic information. Blood chemistry samples were obtained to evaluate patients for CAD. Mini-Mental State Examination (MMSE) and CANTAB using three subsets including intra-extra dimensional set shift, spatial span (SSP), and pattern recognition memory (PRM) were used to assess participants' cognitive function.

\section{Results}

The SSP and PRM were significantly lower in patients with CAD as compared with healthy controls. There were significant relationships of PRM with creatine kinase- muscle/brain, aspartate aminotransferase, and total cholesterol. On the other hand, SSP was found to have a significant relationship with triglycerides.

\section{Conclusion}

There is cognitive impairment in CAD patients that needs to be assessed for early interventions to maintain cognitive functions.

Received 01/06/2020

Review began 01/14/2020 Review ended 01/18/2020 Published 01/21/2020

(c) Copyright 2020

Balbaid et al. This is an open access article distributed under the terms of the Creative Commons Attribution License CC-BY 3.0., which permits unrestricted use, distribution, and reproduction in any medium, provided the original author and source are credited.
Categories: Neurology

Keywords: coronary artery disease, cognitive impairment, cognitive function

\section{Introduction}

Cardiovascular diseases (CVDs) are a group of diseases that affect the heart or blood vessels [1]. Most heart diseases present in the form of coronary artery disease (CAD), which is a predominant cause of mortality in patients with heart disease [2]. Though death due to CVD is declining, CVD is still the leading cause of noncommunicable disease (NCD) mortality and morbidity worldwide [3]. CVD in the Kingdom of Saudi Arabia (KSA) is a cause of the majority of deaths, exceeding untreatable fatal diseases such as cancer [4]. According to a World Health Organization (WHO) report, 644 in 100,000 people aged 30-70 years died due to NCD, 62\% (401 per 644) of which were due to CVD and diabetes in KSA [4]. This raises a huge concern for health care institutions to develop health protocols to detect heart problems before they reach an advanced stage. CAD or ischemic heart disease (IHD) is a common form of CVD that is caused by an obstruction or disruption of coronary blood flow usually due to an advanced atherosclerotic lesion that will eventually lead to a disorder known as myocardial infarction or ischemia [5]. The prevalence of CAD in KSA is 5.5\% [6]. Recently, it has been shown that there is a possible correlation between $\mathrm{CAD}$ and impaired cognitive function, which affects the mental capacity and health status of CAD patients [7]. Recent studies have reported a marked decrease in regional brain volume in patients with CAD accompanied by significant cerebral atrophy, hypoperfusion, and white matter disease in the brains of older adults with an increased risk of vascular disease [7-8]. Although these observations suggest a possible link between CAD and cognitive decline or neurological dysfunction, the interaction of age, vascular disease, and cerebrovascular dysfunction are complex and not well understood. 
Depression is the most common comorbid psychiatric disorder in patients with chronic illness such as CAD and chronic obstructive pulmonary disease, with rates consistently found to be two to three times higher than those in the general population [9]. The prevalence of depression is twice as higher in women than in men [10]. About $17-27 \%$ of patients with CAD have major depression [11]. Although the presence of a relationship between depression and cardiac disease is obvious, the mechanisms underlying this remain unclear.

Some studies have hypothesized underlying mechanisms such as neuroendocrine dysfunction, while also suggesting other important factors such as smoking history, sedentary lifestyle, and a delay in seeking treatment [12-13]. According to the WHO, overweight and obesity are defined as excessive fat accumulation that may impair body function and ultimately health status. In 2014, more than 1.9 billion adults aged 18 years and older were overweight, with over 600 million of these adults being obese [14]. A recent study among university students in KSA has reported a 36.8\% prevalence of obesity and overweight [15]. Other studies also found that obesity is associated with an increased risk of CVD and type 2 diabetes [16]. Some suggest that obese patients have a higher risk of morbidity and mortality [17]. Furthermore, obesity is the most prevalent risk factor for CAD [18]. Some studies have shown that elevated levels of adipokines are linked to increased body mass index (BMI) [19]. Adipose tissue secretes hormones, peptides, and other molecules that may potentially act as pro-atherogenic markers [20].

This study was conducted to evaluate the cognitive impairment hypothesis in patients with CAD and aims to provide better preventive strategies in the near future. This study includes mental status evaluation along with lab investigations and imaging studies to elucidate the relationship between cognitive impairment and CAD severity in middle-aged patients in KSA.

\section{Materials And Methods \\ Participants}

Study Design, Setting, and Sampling Technique

An observational case-control study was performed at the Departments of Physiology and Cardiac Sciences, College of Medicine, King Khalid University Hospital, Riyadh, KSA. A non-probability quota sampling technique was used to recruit our participants (57 patients with CAD and 60 healthy controls). Participants were included or excluded as per the selection criteria.

Inclusion and Exclusion Criteria

Inclusion in the patient group required participants to be diagnosed with CAD based on symptoms, medical history, and risk factors. Participants had to be aged between 30 and 60 years. Patients with any history of neurological or psychological disorders such as Alzheimer's disease, depression, cognitive impairment, anxiety, transient ischemic attack, and stroke were excluded. Participants were also excluded if they presented with any disorders that may affect the brain, such as thyroid disorders, chronic liver disease, chronic kidney disease, and acute diabetic states such as diabetic ketoacidosis. The participants in the healthy control group were interviewed to confirm that there were free of cardiovascular illness, medications, and significant underlying neurological or psychiatric illness, and had no history of head injury or drug/alcohol abuse that would potentially affect their cognitive function.

\section{Instruments used}

Assessment of Participants' Baseline Information

Self-administered questionnaires were used to collect data about participants' demographic information. Blood chemistry samples were also obtained to evaluate patients for CAD. Venous blood samples were analyzed for brain natriuretic peptide, creatine kinase-muscle/brain (CKMB), alanine aminotransferase, aspartate aminotransferase (AST), total cholesterol (TC), low-density lipoprotein, highdensity lipoprotein, and triglycerides (TGs).

\section{Mini-Mental State Examination}

The Mini-Mental State Examination (MMSE) is one of the most widely used tools for quantitative assessment of cognitive function. The test consists of 11 questions assessing various cognitive functions, including two questions concerning orientation, one concerning registration, one concerning memory, five concerning language, one concerning attention and calculation, and one concerning visual construction [5]. The MMSE takes 5-10 minutes to administer, making it a practical tool for research purposes. The test has a maximum score of 30, with a score below 23 being indicative of cognitive impairment. The standard MMSE is in English and was used with English-speaking participants [5]. For Arabic speakers, a translated version of the MMSE was used; this version was introduced in 1999 by a group of Saudi researchers [6]. 
The Cambridge Neuropsychological Test Automated Battery (CANTAB) test was used utilizing the following three subtests [8]:

1. Intra-extra dimensional set shift (IED): IED set shift is a test of rule acquisition and reversal. Stimuli consisting of two potential dimensions, color-filled shapes and white lines, were used in the test. Simple stimuli consisted of just one of these dimensions, whereas compound stimuli consisted of both, namely white lines overlying color-filled shapes. The participants started by seeing two simple color-filled shapes. They then had to learn which one was correct by selecting a stimulus and then receiving a feedback, which allows the participant to know which stimulus is correct. After six correct responses, the stimuli and/or rules are changed. These shifts are initially intra-dimensional (e.g. color-filled shapes remain the only relevant dimension) and later extra-dimensional (white lines become the only relevant dimension). Participants progress through the test by satisfying a set criterion of learning at each stage (six consecutive correct responses). If at any stage the participant fails to reach this criterion after 50 trials, the test terminates.

2. Spatial span (SSP): the SSP assesses working memory capacity using white squares that are displayed on a screen, some of which briefly change color in a variable sequence. The participant must then select the boxes which changed color in the same order in which they were displayed by the computer (for the forward variant) or in the reverse order (for the backward variant). The number of boxes in the sequence increases from two at the start of the test to nine at the end of it. The sequence and colors are varied throughout the test.

3. Pattern recognition memory (PRM): the PRM is a measure of visual PRM tested in a two-choice forced discrimination paradigm. The participants were presented with a series of visual patterns, one at a time, in the middle of the screen. These patterns were designed such that they cannot be easily given verbal labels. In the recognition phase, the participants were required to choose between a pattern they had been shown and a novel pattern. This was repeated with new patterns. The second recognition phase was given immediately or after a delay.

\section{Statistical analysis}

Data were analyzed using SPSS for Windows, Version 21.0 (IBM Corp., Armonk, NY). Categorical data are expressed as absolute numbers and percentages. Numeric data are summarized as mean, median, standard deviation (SD), and range. Categorical variables were compared using the chi-square test. Student's t-test was used for normally distributed data, and the Mann-Whitney U test for non-normally distributed data. Spearman's rank order and Pearson correlations were used to assess the association between variables. Twotailed P-values less than 0.05 were considered statistically significant.

\section{Results}

\section{Sample characteristics}

The patient group ( $n=57$ ) had a mean age of $48.63 \pm 8.42$ years, whereas the control group $(n=60)$ had a mean age of $46.37 \pm 7.46$ years. The difference in mean age between the two groups was not statistically significant $(p=.130)$. There was a significant difference in MMSE score among two groups $(p=.043)$. The demographic data of the participants are summarized in Table 1.

\begin{tabular}{|c|c|c|c|}
\hline Variables & Patients $(n=57)$ & Healthy Control $(n=60)$ & P-Value \\
\hline Age & $48.63 \pm 8.42$ & $46.37 \pm 7.46$ & .130 \\
\hline Body mass index & $28.12 \pm 4.62$ & $28.24 \pm 4.51$ & .910 \\
\hline Mini-Mental State Examination & $27.46 \pm 2.12$ & $28.26 \pm 1.54$ & .043 \\
\hline
\end{tabular}

TABLE 1: Characteristics of study patients with coronary artery disease and healthy control.

\section{Cognitive function}

There was no significant difference in IED total between the patient and control groups ( $\mathrm{p}=.293$ ) (Table 2). Additionally, there was no significant difference between the two groups in the number of IED stages completed ( $\mathrm{p}=.413)$. However, there was a significant difference in PRM $(\mathrm{p}=.002)$ and SSP $(\mathrm{p}=.000)$ results between patients and controls. 


\section{Cureus}

\begin{tabular}{|c|c|c|c|}
\hline Variables & Patients, Mean \pm SD & Healthy Control, Mean \pm SD & P-Value \\
\hline IED total errors (adjusted) & $23.50 \pm 41.36$ & $14.97 \pm 20.06$ & .293 \\
\hline IED stages completed & $8.55 \pm 1.82$ & $8.84 \pm .884$ & 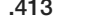 \\
\hline PRM percent correct & $71.04 \pm 13.29$ & $81.53 \pm 13.95$ & .002 \\
\hline SSP length & $4.76 \pm 1.47$ & $6.03 \pm 1.77$ & .000 \\
\hline
\end{tabular}

TABLE 2: Comparison of cognitive functions between coronary artery disease patients and healthy control.

SD, standard deviation; IED, Intra-extra dimensional set shift; PRM, pattern recognition memory; SSP, spatial span

Table 3 shows the significant relationships of PRM with CKMB ( $\mathrm{r}=.526 ; \mathrm{p}=0.025)$, AST ( $\mathrm{r}=.371 ; \mathrm{p}=0.031)$, and $\mathrm{TC}(\mathrm{r}=.420 ; \mathrm{p}=0.019)$. On the other hand, SSP was found to be significantly associated with TG $(\mathrm{r}=.421$; $\mathrm{p}=0.036)$.

\begin{tabular}{|l|l|l|l|l|}
\hline & IED Total Errors (Adjusted) & IED Stages Completed & PRM Percent Correct & SSP Length \\
\hline BNP & -.082 & .400 & -.284 & .053 \\
CKMB & -.036 & .159 & $.526^{*}$ & -.040 \\
ALT & .014 & .211 & .320 & .199 \\
AST & .228 & -.035 & $.371^{*}$ & -.005 \\
LDH & -.108 & .166 & .072 & .250 \\
TC & .163 & -.342 & $.420^{*}$ & .264 \\
\hline HDL & .166 & -.321 & -.055 & .037 \\
LDL & .014 & -.103 & -.027 & .009 \\
TG & .033 & -.337 & .312 & $.421^{*}$ \\
\hline
\end{tabular}

\section{TABLE 3: Correlation of cognitive task and blood marker in coronary artery disease patients.}

BNP, brain natriuretic peptide; CKMB, creatine kinase-muscle/brain; ALT, aminotransferase; AST, aspartate aminotransferase; LDH, lactate dehydrogenase; TC, total cholesterol; HDL, high-density lipoprotein; LDL, low-density lipoprotein; TG, triglycerides

${ }^{*}$ Correlation is significant at the 0.05 level

\section{Discussion}

The prevalence of cognitive impairment is very high in CAD patients and is approximately $35 \%$ [21]. These figures are alarming and need further exploration of possible causes of cognitive decline in such cases. Similar to the results of our study, cognitive decline in memory pattern tested by PRM and SSP has been reported in CVD patients, which is aggravated after cardiac catheterization. The possible reasons could be ischemic insults that the brain receives during such events [22]. Another possible reason for cognitive decline in CVD patients may be the use of cardiovascular drug that can lead to cognitive decline. The new point raised in our study is that we assessed cognition through a standard computer software CANTAB (Cambridge Cognition Ltd., Cambridge, UK) [23-24].

In a recent report, a standardized battery of tests recommended by the National Institute of Neurological Disorders and Stroke-Canadian Stroke Network for the investigation of vascular cognitive impairment and dementia was used to assess cognitive performance. The results were similar to those of our study in that patients with CAD and endothelial dysfunction had poor verbal memory and that improvement in endothelial function during treatment was significantly associated with improvements in overall cognition and processing speed [25]. 
Another study reported that poorer peak oxygen uptake is associated with poorer cognition, particularly executive function, in patients with CAD independent of other cardiac risk

factors. Cardiopulmonary fitness may be a protective factor for cognition in patients with CAD [26]. Our data indicate the importance of neurocognitive assessments in CVD patients and opens doors for the beneficial effects of lifestyle modifications and pharmacotherapy in CVD.

\section{Conclusions}

The presence of cognitive impairment among CAD patients is an important problem and needs to be recognized to allow for interventions to maintain cognitive functions. Early memory assessment in patients could help the timely introduction of treatments, including memory training, and could be used as a tool for classifying symptoms indicating disease deterioration.

\section{Additional Information \\ Disclosures}

Human subjects: Consent was obtained by all participants in this study. King Saud University College of Medicine Institutional Review Board issued approval E-13-983. Animal subjects: All authors have confirmed that this study did not involve animal subjects or tissue. Conflicts of interest: In compliance with the ICMJE uniform disclosure form, all authors declare the following: Payment/services info: All authors have declared that no financial support was received from any organization for the submitted work. Financial relationships: All authors have declared that they have no financial relationships at present or within the previous three years with any organizations that might have an interest in the submitted work. Other relationships: All authors have declared that there are no other relationships or activities that could appear to have influenced the submitted work.

\section{References}

1. Noels H, Bernhagen J: The CXCR4 ligand/receptor family and the DPP4 protease in high-risk cardiovascular patients. Front Immunol. 2016, 7:58. Accessed: January 21, 2020: 10.3389/fimmu.2016.00058

2. Mozaffarian D, Benjamin EJ, Go AS, et al.: Executive summary: heart disease and stroke statistics-2015 update: a report from the American Heart Association. Circulation. 2015, 131:434-441. 10.1161/CIR.0000000000000157

3. Roth GA, Huffman MD, Moran AE, Feigin V, Mensah GA, Naghavi M, Murray CJ: Global and regional patterns in cardiovascular mortality from 1990 to 2013. Circulation. 2015, 132:1667-1678. 10.1161/CIRCULATIONAHA.114.008720

4. Al-Nozha MM, Arafah MR, Al-Mazrou YY, et al.: Coronary artery disease in Saudi Arabia . Saudi Med J. 2004, 25:1165-1171.

5. Folstein MF, Folstein SE, McHugh PR: “Mini-mental state”: a practical method for grading the cognitive state of patients for the clinician. J Psychiatr Res. 1975, 12:189-198. 10.1016/0022-3956(75)90026-6

6. Wrobel NH, Farrag MF: A preliminary report on the validation of the geriatric depression scale in Arabic . Clin Gerontol. 2006, 29:33-46. 10.1300/J018v29n04_03

7. Zheng L, Mack WJ, Chui HC, et al.: Coronary artery disease is associated with cognitive decline independent of changes on magnetic resonance imaging in cognitively normal elderly adults. J Am Geriatr Soc. 2012, 60:499-504. 10.1111/j.1532-5415.2011.03839.x

8. Fray PJ, Robbins TW, Sahakian BJ: Neuorpsychiatyric applications of CANTAB. Int J Geriatr Psychiatry. 1999, 11:329-336. Accessed: November 28, 2017: https://onlinelibrary.wiley.com/doi/pdf/10.1002/(SICI)10991166(199604)11:4\%3C329::AID-GPS453\%3E3.0.CO;2-6.

9. Gorman JM: Gender differences in depression and response to psychotropic medication . Gend Med. 2006;3, 93-109. 10.1016/S1550-8579(06)80199-3

10. Rudisch B, Nemeroff CB: Epidemiology of comorbid coronary artery disease and depression. Biol Psychiatry. 2003, 54:227-240. 10.1016/S0006-3223(03)00587-0

11. Carney RM, Freedland KE, Veith RC: Depression, the autonomic nervous system, and coronary heart disease . Psychosom Med. 2005, 67:29-33. 10.1097/01.psy.0000162254.61556.d5

12. Friedman JI, Tang CY, de Haas HJ, et al.: Brain imaging changes associated with risk factors for cardiovascular and cerebrovascular disease in asymptomatic patients. JACC Cardiovasc Imaging. 2014, 7:1039-1053. 10.1016/j.jcmg.2014.06.014

13. Anazodo UC, Shoemaker JK, Suskin N, St Lawrence KS: An investigation of changes in regional gray matter volume in cardiovascular disease patients, pre and post cardiovascular rehabilitation. Neuroimage Clin. 2013, 3:388-395. Accessed: January 21, 2020: 10.1016/j.nicl.2013.09.011

14. Scott KM, Hwang I, Chiu WT, et al.: Chronic physical conditions and their association with first onset of suicidal behavior in the world mental health surveys. Psychosom Med. 2010, 72:712-719. 10.1097/PSY.0b013e3181e3333d

15. Hamam FA, Eldalo AS, Alnofeie AA, Alghamdi WY, Almutairi SS, Badyan FS: The association of eating habits and lifestyle with overweight and obesity among health sciences students in Taif University, KSA. J Taibah Univ Med Sci. 2017, 12:249-260. Accessed: January 21, 2020: 10.1016/j.jtumed.2016.12.001

16. WHO: Obesity and overweight. (2016). Accessed: November 5, 2017: http://www.who.int/mediacentre/factsheets/fs311/en/.

17. Musaiger AO, Al-Mannai M, Al-Haifi AR, et al.: Prevalence of overweight and obesity among adolescents in eight Arab countries: comparison between two international standards (ARABEAT-2). Nutr Hosp. 2016, 33:567. 10.20960/nh.567

18. Wang Y, Rimm EB, Stampfer MJ, Willett WC, Hu FB: Comparison of abdominal adiposity and overall obesity 


\section{Cureus}

in predicting risk of type 2 diabetes among men. Am J Clin Nutr. 2005, 81:555-563. 10.1093/ajcn/81.3.555

19. Adams KF, Schatzkin A, Harris TB, et al.: Overweight, obesity, and mortality in a large prospective cohort of persons 50 to 71 years old. N Engl J Med. 2006, 355:763-778. 10.1056/NEJMoa055643

20. Yusuf S, Hawken S, Ounpuu S, et al.: Obesity and the risk of myocardial infarction in 27,000 participants from 52 countries: a case-control study. Lancet. 2005, 366:1640-1649. 10.1016/S0140-6736(05)67663-5

21. Silbert BS, Scott DA, Evered LA, Lewis MS, Maruff PT: Preexisting cognitive impairment in patients scheduled for elective coronary artery bypass graft surgery. Anesth Analg. 2007, 104:1023-1028. 10.1213/01.ane.0000263285.03361.3a

22. Whitlock EL, Diaz-Ramirez LG, Smith AK, Boscardin WJ, Avidan MS, Glymour MM: Cognitive change after cardiac surgery versus cardiac catheterization: a population-based study. Ann Thorac Surg. 2019, 107:11191125. 10.1016/j.athoracsur.2018.10.021

23. Svahn S, Lövheim H, Isaksson U, Sandman PO, Gustafsson M: Cardiovascular drug use among people with cognitive impairment living in nursing homes in northern Sweden. Eur J Clin Pharmacol. 2020, 10.1007/s00228-019-02778-y

24. Lanctôt KL, O'Regan J, Schwartz Y, Swardfager W, Saleem M, Oh PI, Herrmann N: Assessing cognitive effects of anticholinergic medications in patients with coronary artery disease. Psychosomatics. 2014, 55:61-68. 10.1016/j.psym.2013.04.004

25. Saleem M, Herrmann N, Dinoff A, et al.: Association between endothelial function and cognitive performance in patients with coronary artery disease during cardiac rehabilitation. Psychosom Med. 2019, 81:184-191. 10.1097/PSY.000000000000065

26. Swardfager W, Herrmann N, Marzolini S, et al.: Cardiopulmonary fitness is associated with cognitive performance in patients with coronary artery disease. J Am Geriatr Soc. 2010, 58:1519-1525. 10.1111/j.15325415.2010.02966.x 